Research Paper

\title{
Potential Dual Immunomodulatory Role of VEGF in Ulcerative Colitis and Colorectal Carcinoma
}

\author{
Natasa D Zdravkovic ${ }^{1}$, Ivan P Jovanovic ${ }^{\circledR}$, Gordana D Radosavljevic ${ }^{2}$, Aleksandar N Arsenijevic ${ }^{2}$, Nebojsa \\ D Zdravkovic ${ }^{3}$, Slobodanka Lj Mitrovic ${ }^{4}$, Nebojsa N Arsenijevic ${ }^{2}$ \\ 1. Center for Gastroenterology, Clinical Center Kragujevac, Serbia \\ 2. Center for Molecular Medicine and Stem Cell Research, Faculty of Medical Sciences, University of Kragujevac, Serbia \\ 3. Department of Medical informatics and statistics, Faculty of Medical Sciences, University of Kragujevac, Serbia \\ 4. Department of Pathology, Faculty of Medical Sciences, University of Kragujevac, Serbia
}

$\triangle$ Corresponding author: Ivan Jovanovic, MD, PhD, Center for Molecular Medicine and Stem Cell Research, Faculty of Medical Sciences University of Kragujevac, Svetozara Markovica 69, 34000 Kragujevac, Serbia. Tel +38134306800; Fax. +38134306800112; E-mail: ivanjovanovic77@gmail.com

() Ivyspring International Publisher. This is an open-access article distributed under the terms of the Creative Commons License (http://creativecommons.org/ licenses/by-nc-nd/3.0/). Reproduction is permitted for personal, noncommercial use, provided that the article is in whole, unmodified, and properly cited.

Received: 2013.12.03; Accepted: 2014.05.29; Published: 2014.07.02

\begin{abstract}
Objective. Progression from ulcerative colitis (UC) toward colorectal carcinoma (CRC) is multistep process that includes gene alterations of tumor suppressor genes, such as $\mathrm{p} 53$ and $\mathrm{p} / 6$. The aim of this study was to investigate the expression patterns of p $16, p 53$ and VEGF in affected tissue and serum levels of cytokines TNF- $\alpha$, IFN-y, IL-4, IL-6, IL-I0 and IL-I7 in patients with UC and CRC, respectively.

Matherials and methods. Serum levels of cytokine in patients with UC $(n=24)$ and CRC $(n=75)$ and in a healthy group $(n=37)$ were analyzed by ELISA. Endoscopic biopsies specimens of $U C$ and CRC were studied by immunohistochemical staining for pl6, p53 and VEGF.

Results. Patients with UC with presence of extraintestinal manifestations, complications, and positive staining of p 16, p53 and VEGF respectively had higher serum levels of pro-inflammatory cytokines. Higher percentage of CRC patients had positive staining of pl6, p53 and VEGF. CRC patients with positive staining of VEGF had decreased systemic values of pro-inflammatory IFN- $\gamma$ and increased values of immunosuppressive IL- 10 .

Conclusions. Relatively low IL- 10 in patients with severe UC is insufficient to compensate IL-6 secretion and subsequently enhanced type I/I7 immune response. In UC patients, p/6 and p53 induce enhanced VEGF expression and subsequent production of pro-inflammatory TNF- $\alpha$ and IL-6. In CRC patients VEGF seems to have immunosuppressive role. It appears that tumor suppressor gene-VEGF axis have dual role on immune response in inflammation of UC and tumor growth and progression of CRC.
\end{abstract}

Key words: ulcerative colitis, colorectal carcinoma, VEGF, Th1, Th17

\section{Introduction}

Inflammatory bowel disease (IBD) presents a chronic, relapsing and progressive inflammation of gastrointestinal tract, which includes two major entities: ulcerative colitis (UC) and Crohn`s disease (CD). Ulcerative colitis is inflammatory disease affecting distal colon and rectum, limited to the mucosa [1].

The immune system seems to be major mediator in UC pathogenesis and progression [2]. Immune system is consisted of innate and acquired immunity.
The innate immunity consist of monocyte/ macrophages, neutrophils and NK cells, while main components of acquired immunity are B lymphocytes (humoral immunity) and $\mathrm{T}$ lymphocytes (cellular immunity), which can be further divided on $\mathrm{CD} 4^{+}$ helper and $\mathrm{CD}^{+}$cytotoxic $\mathrm{T}$ lymphocytes [3]. Beside monocyte/macrophages, $\mathrm{CD} 4^{+}$helper $\mathrm{T}$ lymphocytes are major producers of cytokines and can be classified according to type of cytokines they produce: Th1 
(IFN- $\gamma$ and TNF- $\alpha$ ), Th2 (IL-4, IL-5 and IL-13), Th17 (IL-6 and IL-17) and Tregs (IL-10 and TGF- $\beta$ ) [3].

Ulcerative colitis is an inflammatory disorder that predisposes development of CRC. In fact, about one-third of all CRCs are associated with chronic IBD [4-6]. Progression from UC toward CRC is multistep process that includes dysplasia, high grade dysplasia and cancer [1]. Molecular abnormalities that underline this process are consequence of chromosomal instability and gene alterations. Mutations of tumor suppressor genes, such as p53 and p16 are among the major gene alterations in oncogenesis [7].

Tumor suppressor gene, p16 (INK4A) is upregulated by the CDKN2A protein [8]. The main role of p16 is formation of inhibitory cdk4-6/p16 complexes, degradation of free Cyclin $\mathrm{D}$ by an ubiquitin-dependent proteasome pathway and subsequent inhibition of cell cycle progression [9]. Besides its role in cancer as an inhibitor of cell-cycle progression, p16 plays important role in modulation of immune response [10]. In response to DNA damage, wild-type p53 upregulates p21 (WAF1/Cip1) protein, which is a general inhibitor of CDKs and contributes to G1 cell cycle arrest under these circumstances [11]. Many authors have reported p53 mutations in epithelium adjacent to dysplasia in UC [12-15]. These alterations of p53 underlie multistep process of oncogenesis [16]. Vascular endothelial growth factor (VEGF) belongs to a family of platelet-derived growth factors and its involvement in angiogenesis in various tumors is well described [17]. Still, role of VEGF in modulation of immune response remains unclear.

Therefore, the aim of this study was to investigate the expression patterns of p16, p53 and VEGF in affected tissue and serum levels of cytokines TNF- $a$, IFN-ץ, IL-4, IL-6, IL-10 and IL-17 in patients with UC and $\mathrm{CRC}$, respectively. We report higher values of pro-inflammatory cytokines in patients with UC with presence of extraintestinal manifestations, complications, and positive staining of p16, p53 and VEGF, respectively. We also assessed the associations of decreased systemic values of pro-inflammatory IFN- $\gamma$ and increased values of immunosuppressive IL-10 in CRC patients with positive staining of VEGF. Expression of p53 is a found to be highly specific marker in differentiation between UC and CRC.

\section{Material and methods}

\section{Patients}

The study was conducted at Center for Gastroenterology, Clinical Centre of Kragujevac and Center for Molecular Medicine and Stem Cell Research, Faculty of Medical Sciences, University of Kragujevac, Serbia, between May 2011 and March 2013. All of the patients gave their informed consent and research project was approved by two Ethics Committees of these institutions.

We investigated total of 75 patients with colorectal cancer (41 males and 34 females; mean age: $64.89 \pm 10.51$ years) and 24 patients with ulcerative colitis (13 males and 11 females; mean age: $45.75 \pm 19$ years). The diagnosis of colorectal carcinoma and ulcerative colitis was based on endoscopic and histopathological criteria. The patients with ulcerative colitis were classified into three groups, according to histological grade: grade I- diffuse mucosal inflammation with only mild or moderate crypt distortion, mucosal atrophy or mucus depletion; grade II- acute inflammation with severe crypt distortion and diffuse goblet cell depletion (mucus depletion); inflammation is diffuse and solely mucosal; vascularity is increased; grade III- diffuse acute and chronic inflammation with increased vascularity but little mucus depletionsuggesting resolving phase [18]. The patients with CRC were classified into four groups according to histological grade: grade I- well differentiated, grade II- moderately differentiated, grade III- poorly differentiated and grade IV- undifferentiated [19].

The study did not include patients with previously diagnosed colorectal cancer patients who underwent surgery or were treated with radiation and chemotherapy, as well as patients with ulcerative colitis who were previously treated with antibiotics, aminosalicylates, corticosteroids, immunosuppressive agents, and biological therapy. All subjects had a complete medical history, including physical examination, routine laboratory tests and diagnostic imaging (chest X-ray, abdominal ultrasound, abdominal computed tomography scan and endoscopy).

\section{Assessment of serum TNF- $\alpha$, IL-6, IL-I7, IFN- Y, IL-4, IL-I 0 and CRP level}

The blood samples of all patients were collected before application of therapy. The control group consisted of 37 healthy individuals. Control subjects were selected from volunteer blood donors at the Clinical Centre of Kragujevac. A control group was matched with the experimental groups on the basis of gender. Blood samples were collected from each studied subject; blood clot was cut and centrifuged for separating the serum and all serum samples were kept at $-20^{\circ} \mathrm{C}$ before use. Repeated freeze-thaw cycles were avoided to prevent loss of bioactive substances.

Serum levels of cytokines were measured, as described before [20] using sensitive enzyme-linked immunosorbent assay (ELISA) kits (R\&D Systems Minneapolis, MN for IL-4, IL-6, TNF-a, CRP, IL-10 and IL-17; BD Biosciences San Diego, CA for IFN- $\gamma$ ) 
specific for the human cytokines, according to the manufacturer's instructions.

\section{Immunohistochemical studies of VEGF, p I 6 and p53}

Immunohistochemical staining of VEGF, p16 and p53 protein was performed using streptavidin-biotin technique. Immunohistochemistry was performed on multiple endoscopics colonic mucosal biopsy specimens collected from patients with ulcerative colitis and endoscopics colonic biopsy specimens from patients with colorectal carcinoma. The sections (4-5 $\mu \mathrm{m})$ of formalin-fixed, paraffin-embedded tissue samples were de-paraffinized with xylene and them rehydrated in a series of $100 \%, 96 \%, 70 \%$ and $50 \%$ alcohol. Briefly, antigen retrieval was performed by microwave heating for 20 minutes in $10 \mathrm{mM}$ sodium citrate buffer ( $\mathrm{pH}$ 6.0). The sections were incubated with $3 \%$ hydrogen peroxide to block endogenous peroxidase activity and them incubated with mouse monoclonal antibodies against VEGF (ab16883, Abcam, Cambridge, UK, at a 1:200 dilution), p53 (ab17869-250, Abcam, Cambridge, UK, at a 1:200 dilution) and p16 (sc-81156, Santa Cruz Biotechnology, Santa Cruz, CA, USA, at a 1:50 dilution) for $60 \mathrm{~min}$ in a humid chamber, respectively. All primary antibodies were diluted in 1\% BSA/PBS. After the primary antibody, biotinylated secondary antibodies were applird, followed by detection using the ABC (Avidin-Bioton peroxidase Complex) method. Diaminobenzedine was used as the chromogen. Light counterstaining was performed with haematoxylin. The slides were examined by conventional light microscopy. Negative controls were treated identically with the primary antibodies omitted. Positive controls consisted of tissue known to contain the protein of interest.

The VEGF, p16 and p53 stained sections were assessed semiquantitatively by two pathologists. The staining score was evaluated as the percentage of stained cells out of the total number of evaluated cells. The extent of each staining pattern of p16 and p53 was recorded as the number of positive nuclei per 250 cells. The same pattern was used for VEGF, in cytoplasm. Percentage of positive cells was determined by counting 5 non overlapping microscopic fields at an x400 magnification. Staining for p16, p53 and VEGF was defined as positive when $>10 \%$ of the cells were stained and negative when $\leq 10 \%$ of the cells were stained $[21,22]$.

\section{Statistical analysis}

Software package IBM SPSS Statistics 20 was used for the statistical analysis. Data are grouped and shown in tables and graphs. Methods of descriptive statistics were used in determining measures of central tendency and variability. The results are presented as mean and standard deviation. Pearson's and Spearman's correlation coefficients were used for determining the strength and direction of correlation between the variables.

Chi-squared test was used for determining the independence of variables. In determining statistically significant difference between the means of two groups Student's t-test for independent samples was used if the data were normally distributed, while Mann-Whitney U-test was used for data that were not normally distributed. One-Way ANOVA test was used for determining statistically relevant differences between the means of various groups of normally distributed data and Kruskal-Wallis test for data that were not normally distributed. In determining the normality of data distribution Kolmogorov-Smirnov test and Shapiro-Wilk test were used. Levene's test for equality of variances was used to determine the homogenity of variances. This test determines if the results from two groups of data have equal variances. ROC curve was used for the review of sensitivity and specificity. All statistical analyses in this paper were conducted with confidence interval of $95 \%$. The results of statistical analysis were taken as statistically significant if the level of probability of null hypothesis is lower than $5 \%$, that is to say, if the relevance of the test is $\mathrm{p}<0.05$.

\section{Results}

\section{Association between p 16, p53 and VEGF expression and clinicopathological parameters of patients with ulcerative colitis (UC)}

Twenty-four patients with ulcerative colitis, 13 males and 11 females with mean age $45.75 \pm 19$ years were studied. Clinical and pathologic characteristics of these patients are presented in Table 1 . The expression patterns of p16, p53 and VEGF protein were assessed by immunohistochemistry on biopsy specimens. All patients with UC were classified on the basis of positive $(>10 \%$ positive cells) or negative $(\leq 10 \%$ positive cells) immunostaining of p16, p53 and VEGF, respectively. Only nuclear staining of p16 and p53 was scored. More than $10 \%$ of p16 positive cells were detected in $58 \%(14 / 24)$ of patients while only $12.5 \%$ $(3 / 24)$ of patients had positive p53 nuclear staining (Table 1). VEGF expression was found to occur in the cytoplasm and cell membrane. More than $10 \%$ positive cells for VEGF were observed in $37.5 \%(9 / 24)$ of patients with UC.

Further, we found no significant association between expression patterns of p16, p53 and VEGF and disease activity. However, positive staining of 
p16 and VEGF was detected in significantly higher percentages of UC patients with histological grade III $(p=0.026 ; p=0.034$, respectively).

Representative images of immunostaining for with ulcerative colitis are shown in Figure 1.

\section{Serum concentrations of pro- and anti-inflammatory mediators and their ratios in UC patients with different disease activity}

Next, we examined the serum levels of pro-inflammatory (IL-4, IL-6,TNF-a, IFN- $\gamma$ and IL-17) and anti-inflammatory mediators (IL-10) in patients with ulcerative colitis. the mentioned markers examined in tissue of patients

Table I. Clinicopathological features and expression patterns of pl6, p53 and VEGF in patients with UC

\begin{tabular}{|c|c|c|c|c|c|c|}
\hline & p16 $6^{+}$ & p16- & p53+ & p53- & VEGF $^{+}$ & VEGF- \\
\hline & $\begin{array}{c}\mathrm{n}=14 / 24 \\
(\%)\end{array}$ & $\begin{array}{c}\mathrm{n}=10 / 24 \\
(\%)\end{array}$ & $\begin{array}{c}\mathrm{n}=3 / 24 \\
(\%)\end{array}$ & $\begin{array}{c}\mathrm{n}=21 / 24 \\
(\%)\end{array}$ & $\begin{array}{c}\mathrm{n}=9 / 24 \\
(\%)\end{array}$ & $\begin{array}{c}\mathrm{n}=15 / 24 \\
(\%)\end{array}$ \\
\hline \multicolumn{7}{|c|}{ Age (years) } \\
\hline$<40$ & 25.00 & 4.16 & 8.33 & 20.83 & 16.67 & 12.50 \\
\hline$>40$ & 33.33 & 37.25 & 4.17 & 66.67 & 20.83 & 50.00 \\
\hline tp values & \multicolumn{2}{|c|}{0.543} & \multicolumn{2}{|c|}{0.408} & \multicolumn{2}{|c|}{0.443} \\
\hline \multicolumn{7}{|l|}{ Gender } \\
\hline Male & 33.33 & 29.16 & 8.33 & 54.17 & 25.00 & 37.50 \\
\hline Female & 25.00 & 12.50 & 4.17 & 33.33 & 12.50 & 25.00 \\
\hline$p$ values & \multicolumn{2}{|c|}{0.302} & \multicolumn{2}{|c|}{0.806} & \multicolumn{2}{|c|}{0.543} \\
\hline \multicolumn{7}{|c|}{$\begin{array}{l}\text { Disease activ- } \\
\text { ity }\end{array}$} \\
\hline mild & 12.50 & 20.83 & 8.33 & 25.00 & 8.33 & 25.00 \\
\hline moderate & 37.5 & 20.83 & 4.16 & 54.16 & 25.00 & 33.33 \\
\hline severe & 8.33 & 0.00 & 0.00 & 8.33 & 4.17 & 4.17 \\
\hline$p$ values & \multicolumn{2}{|c|}{0.490} & \multicolumn{2}{|c|}{0.306} & \multicolumn{2}{|c|}{0.290} \\
\hline \multicolumn{7}{|c|}{$\begin{array}{l}\text { Histologic } \\
\text { grade }\end{array}$} \\
\hline 1 & 4.17 & 8.33 & 4.17 & 8.33 & 4.17 & 8.33 \\
\hline 11 & 12.50 & 20.83 & 4.17 & 29.16 & 29.17 & 4.17 \\
\hline 111 & 41.67 & 12.50 & 4.17 & 50.00 & 29.17 & 25.00 \\
\hline$p$ values & \multicolumn{2}{|c|}{$* 0.026$} & \multicolumn{2}{|c|}{0.543} & \multicolumn{2}{|c|}{${ }^{*} 0.034$} \\
\hline \multicolumn{7}{|c|}{$\begin{array}{l}\text { Extraintestinal } \\
\text { manifestations }\end{array}$} \\
\hline without & 45.83 & 41.67 & 12.50 & 75.00 & 29.17 & 58.33 \\
\hline with & 12.50 & 0.00 & 0.00 & 12.50 & 8.33 & 4.17 \\
\hline$p$ values & \multicolumn{2}{|c|}{0.340} & \multicolumn{2}{|c|}{0.790} & \multicolumn{2}{|c|}{0.543} \\
\hline \multicolumn{7}{|c|}{ Complications } \\
\hline without & 29.17 & 29.17 & 8.33 & 50.00 & 16.67 & 41.67 \\
\hline with & 29.17 & 12.50 & 4.17 & 37.50 & 20.83 & 20.83 \\
\hline pvalues & \multicolumn{2}{|c|}{0.600} & \multicolumn{2}{|c|}{0.312} & \multicolumn{2}{|c|}{0.569} \\
\hline
\end{tabular}

tp values were assessed by the Kruscal-Wallis method; * $p$-statistically significant. Data are presented as percentages of total number of patients with UC
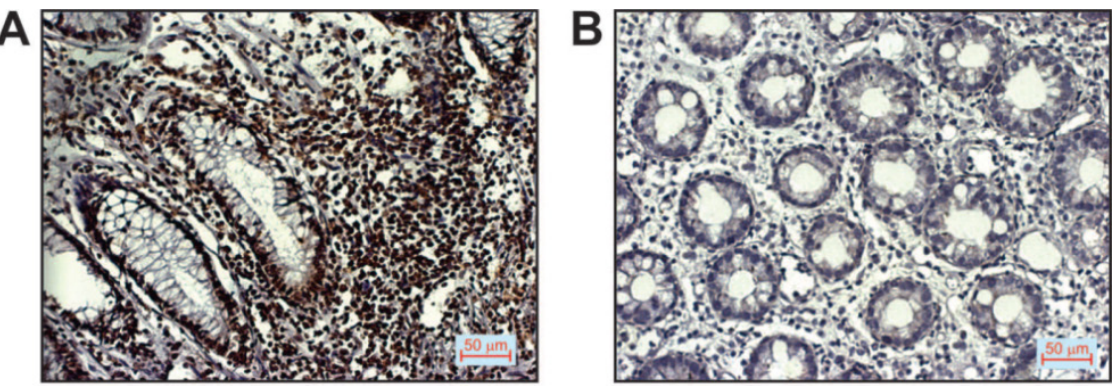

Figure I. p16, p53 and VEGF expression in patients with UC. A. Positive pl6 staining (200x magnification), B. Negative pl6 staining (200x magnification), C. Positive p53 staining (200x magnification), D. Negative p53 staining (200x magnification), E. Positive VEGF staining (200x magnification), $F$. Negative VEGF staining (200x magnification).

Patients with UC were categorized
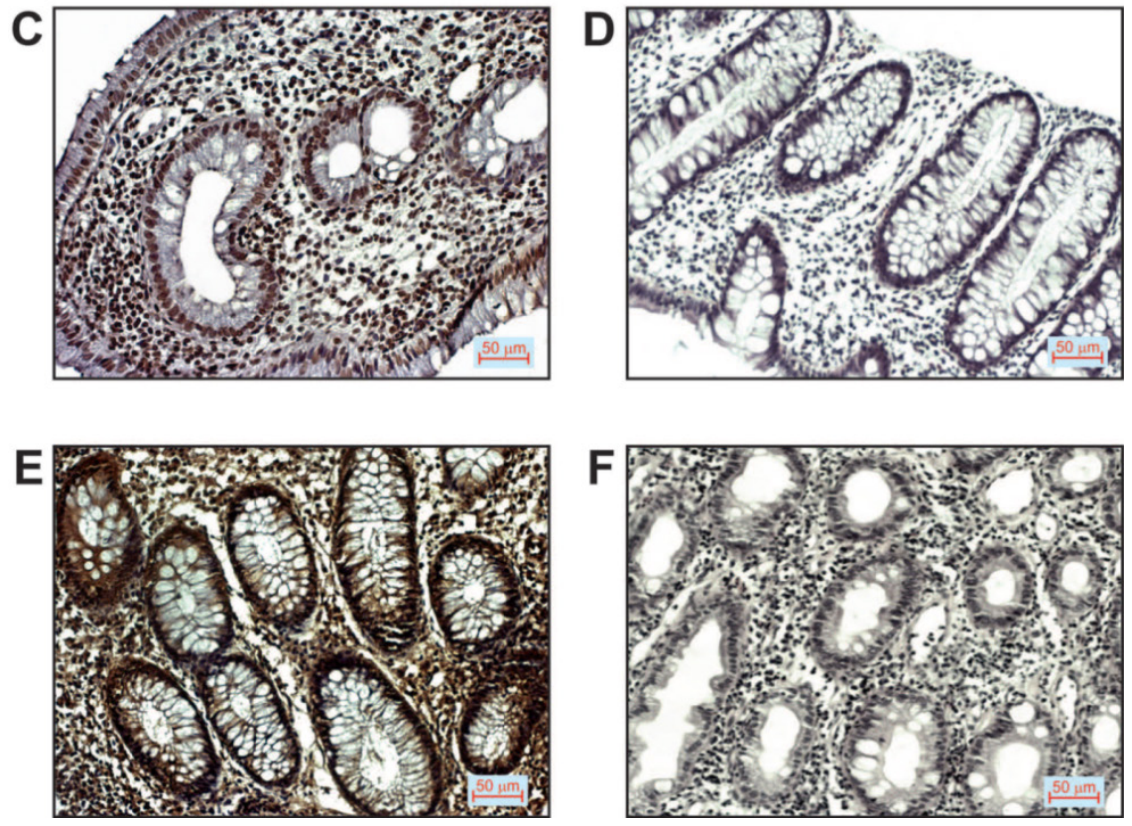

into 3 groups on the basis of clinical activity of disease: patients with mild UC ( $\leq 3$ stools daily with or without blood and had no systemic toxicity), patients with moderate UC (4-6 stools daily with or without blood or had minimal signs of toxicity) and patients with severe UC ( $\geq 7$ stools daily with or without blood, had moderate to severe systemic toxicity: fever, $\geq 10 \%$ weight loss, orthostatic, hemoglobin $\leq 10 \mathrm{~g} / \mathrm{dL}$, or required hospitalization) [23]. We analyzed serum level of CRP in defined groups and found that serum concentration of CRP was significantly higher in patients with severe UC compared to mild UC (202.6 mg/l vs. $25.1 \mathrm{mg} / \mathrm{l}$; $\mathrm{p}=0.005$; data not shown). 
Further, we divided patients in two categories: with extraintestinal manifestations (dermatologic manifestations- pyoderma gangrenosum or erythema nodosum) and without extraintestinal manifestations [24] and analyzed them for systemic values of proand anti-inflammatory mediators and their ratios. We obtained the statistically significant positive correlation between serum level of CRP (not shown) and IL-6, and presence of extraintestinal manifestations $(\mathrm{p}=0.002$; Fig 2A, left panel). It had been suggested that ratio of counterregulatory cytokines could be marker of the disease progress. Therefore, we ana-
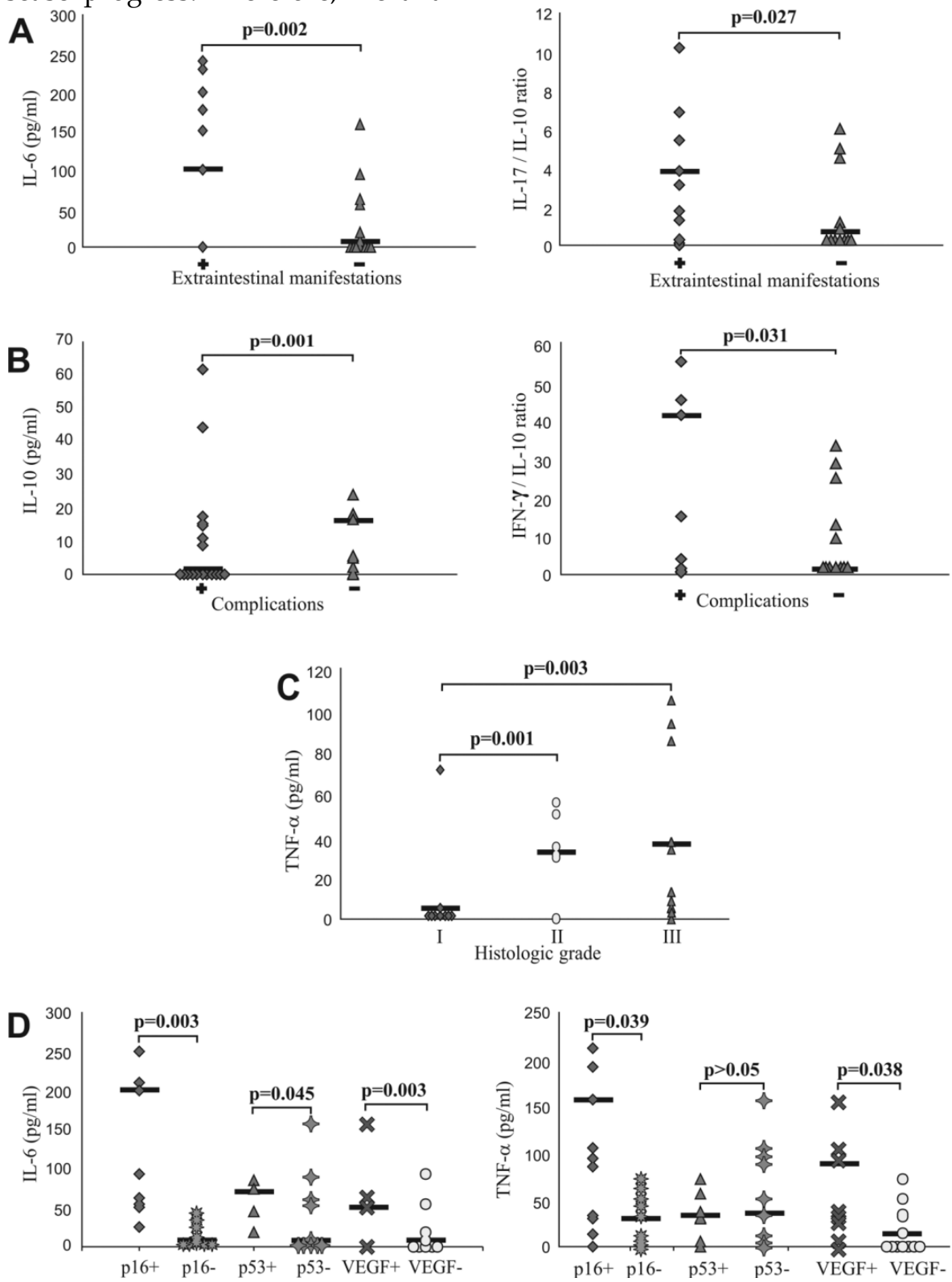

Figure 2. Serum values of mediators of inflammation in patients with UC. A. Increased concentration of IL- 6 and IL-I $7 / I L-I 0$ ratio in patients with UC with presence of extraintestinal manifestations. Patients with UC were divided in two groups, based on presence of extraintestinal manifestations (positive "+" or negative "-"). IL-I7/IL-I0 ratio was evaluated for each patient, separately. B. Decreased concentration of IL-IO and increased IFN-y/IL-IO ratio in patients with UC with presence of complications. Patients with UC were divided in two groups, based on presence of complications (positive "+" or negative "-"). IFN-y/IL- 10 ratio was evaluated for each patient, separately. C. Elevation of TNF- $\alpha$ in sera of patients with higher histological grade. Patients with UC were divided in three groups, according to histological grade. Serum levels of all mentioned cytokines were determined by ELISA. D. Higher serum concentration of TNF- $\alpha$ and IL-6 in patients with positive staining of $p / 6, p 53$ and VEGF. Patients with UC were divided in two groups, based on expression of p 16, p53 and VEGF, respectively. Statistical significance was tested by Mann-Whitney Rank Sum test or independent samples t-test, where appropriate. 
When we divided patients into groups in accordance to histological grade, we found positive correlation between the serum concentration of TNF- $\alpha$ and histological grade. Concentration of TNF- $a$ was significantly lower in patients with histological grade I than in patients with histological grade II $(p=0.001)$, and in patients with histological grade III $(\mathrm{p}=0.003$; Fig 2C).

\section{Higher systemic values of TNF- $\alpha$ and IL-6 in UC patients with positive staining of $\mathrm{pl} 6, \mathrm{p} 53$ and VEGF}

Subjects with UC were classified on the basis of positive or negative immunostaining of p16, p53 and VEGF, respectively and analyzed for systemic values of pro- and anti-inflammatory cytokines. Serum level of TNF-a was significantly higher in group of UC patients with positive staining of $\mathrm{p} 16(\mathrm{p}=0.039)$ and VEGF ( $p=0.038$ ) (Fig 2D, right panel), while serum level of IL-6 was significantly higher in group of UC patients with positive staining of $\mathrm{p} 16(\mathrm{p}=0.003)$, $\mathrm{p} 53$ $(p=0.045)$ and VEGF ( $p=0.003)$ (Fig 2D, left panel). We did not find significant difference for other measured cytokines (data not shown).

\section{Expression of p 16, p53 and VEGF protein in patients with colorectal carcinoma (CRC)}

The next aim of this study was to investigate the expression of p16, p53 and VEGF protein in colorectal carcinoma and to assess the association between their expression and clinico-pathological characteristics of CRC patients. A total of 75 patients with colorectal cancer (41 males and 34 females), of mean age $64.89 \pm$ 10.51 years were studied. Table 2 summarizes the clinical and pathologic characteristics of these patients. The expression patterns of p16, p53 and VEGF protein were assessed on preoperative biopsy specimens of CRC patients. The study subjects were classified based on negative ( $\leq 10 \%$ positive tumor cells) or positive ( $>10 \%$ positive tumor cells) expression of $\mathrm{p} 16$, p53 and VEGF in colorectal carcinoma respectively. Positive p16, p53 and VEGF staining was found in $68.29 \%(28 / 41), 48 \%(36 / 75)$ and $66.67 \%(50 / 75)$ of patients, respectively (Table 2).

Patients with CRC were divided on the basis of positive or negative immunostaining of p16, p53 and VEGF, respectively, as shown in Table 2 . We did not estimated histological grade IV in any of CRC patients. Representative images of immunostaining for the mentioned markers examined in tissue of patients with CRC are shown in Figure 3.

\section{Decreased systemic values of pro-inflammatory IFN-Y and IL-4 and increased values of anti-inflammmatory IL- I 0 in CRC patients with positive staining of VEGF}

Further, we analyzed systemic values of pro- and anti-inflammatory cytokines and their ratios in previously defined groups of patients with CRC. Serum level of IFN- $\gamma$ was significantly lower in group of CRC patients with positive staining of VEGF ( $p=0.032$; Fig 4A), while serum level of IL-10 was significantly higher in same group of patients $(\mathrm{p}=0.045$; Fig 4B). In addition, IFN- $\gamma /$ IL10 ratio was significantly lower in group of CRC patients with positive staining of $\mathrm{p} 16$ $(p=0.025), p 53(p=0.038)$ and VEGF ( $p=0.024$; Fig $4 C)$. We also noticed increased IL-4 serum level in CRC patients with positive staining of $\mathrm{p} 16$ ( $\mathrm{p}=0.021$; Fig $4 \mathrm{D})$ as well as in patients with simultaneous expression of p16 and p53 (p=0.01; Fig 4E).

\section{Higher frequency of patients with CRC had positive staining of p l6, p53 and VEGF, in comparison to patients with UC}

Additionally, we analyzed expression p16, p53 and VEGF between patients with CRC and UC. The frequency of patients with CRC with positive staining for p53 and VEGF, respectively, was significantly higher compared with UC subjects $(p=0.025 ; p=0.035$, respectively) with no significant difference between groups based on p16 staining (Fig 5A).

Table 2. Clinicopathological features and expression patterns of pl6, p53 and VEGF in patients with CRC

\begin{tabular}{|c|c|c|c|c|c|c|}
\hline & $\begin{array}{c}\text { p16 } \\
\text { n=28/ } \\
41(\%)\end{array}$ & $\begin{array}{c}\text { p16 } \\
\mathrm{n}=13 / \\
41(\%)\end{array}$ & $\begin{array}{c}\text { p53 }^{+} \\
\mathrm{n}=36 / \\
75(\%)\end{array}$ & $\begin{array}{c}\text { p53- } \\
\mathrm{n}=39 / \\
75(\%)\end{array}$ & $\begin{array}{c}\text { VEGF }^{+} \\
\mathrm{n}=50 / \\
75(\%)\end{array}$ & $\begin{array}{c}\text { VEGF } \\
\mathrm{n}=25 / \\
75(\%)\end{array}$ \\
\hline \multicolumn{7}{|l|}{$\begin{array}{l}\text { Age } \\
\text { (years) }\end{array}$} \\
\hline$<65$ & 29.20 & 14.63 & 26.66 & 22.66 & 30.66 & 18.66 \\
\hline$>65$ & 39.02 & 17.07 & 21.33 & 30.66 & 36.00 & 14.66 \\
\hline tp values & \multicolumn{2}{|c|}{0.803} & \multicolumn{2}{|c|}{0.706} & \multicolumn{2}{|c|}{0.780} \\
\hline \multicolumn{7}{|l|}{ Gender } \\
\hline Male & 39.02 & 14.63 & 28.00 & 26.66 & 38.66 & 16.00 \\
\hline Female & 29.26 & 17.07 & 20.00 & 25.33 & 28.00 & 17.33 \\
\hline$p$ values & \multicolumn{2}{|c|}{0.132} & \multicolumn{2}{|c|}{0.302} & \multicolumn{2}{|c|}{0.806} \\
\hline \multicolumn{7}{|l|}{ Nuclear grade } \\
\hline 1 & 4.87 & 2.43 & 4,00 & 8.00 & 6.66 & 5.33 \\
\hline 11 & 63.41 & 21.95 & 37,33 & 41.33 & 54.66 & 24.00 \\
\hline lll & 4.87 & 2.43 & 6.66 & 2.66 & 5.33 & 4.00 \\
\hline$p$ values & \multicolumn{2}{|c|}{0.181} & \multicolumn{2}{|c|}{0.453} & \multicolumn{2}{|c|}{0.179} \\
\hline \multicolumn{7}{|l|}{$\begin{array}{l}\text { Histological } \\
\text { grade }\end{array}$} \\
\hline 1 & 34.10 & 17.07 & 18.66 & 30.66 & 34.66 & 14.66 \\
\hline 11 & 31.70 & 7.31 & 26.66 & 18.66 & 30.66 & 14.66 \\
\hline 111 & 2.43 & 7.31 & 2.66 & 2.66 & 4.00 & 1.33 \\
\hline$p$ values & \multicolumn{2}{|c|}{0.321} & \multicolumn{2}{|c|}{0.899} & \multicolumn{2}{|c|}{0.193} \\
\hline \multicolumn{7}{|l|}{ Tumor location } \\
\hline \multicolumn{7}{|l|}{ Rectum } \\
\hline Distal colon & 17.07 & 17.07 & 8.00 & 24.00 & 17.33 & 14.66 \\
\hline Proximal colon & 14.63 & 2.43 & 6.66 & 8.00 & 13.33 & 1.33 \\
\hline$p$ values & \multicolumn{2}{|c|}{0.943} & \multicolumn{2}{|c|}{0.543} & \multicolumn{2}{|c|}{0.612} \\
\hline
\end{tabular}



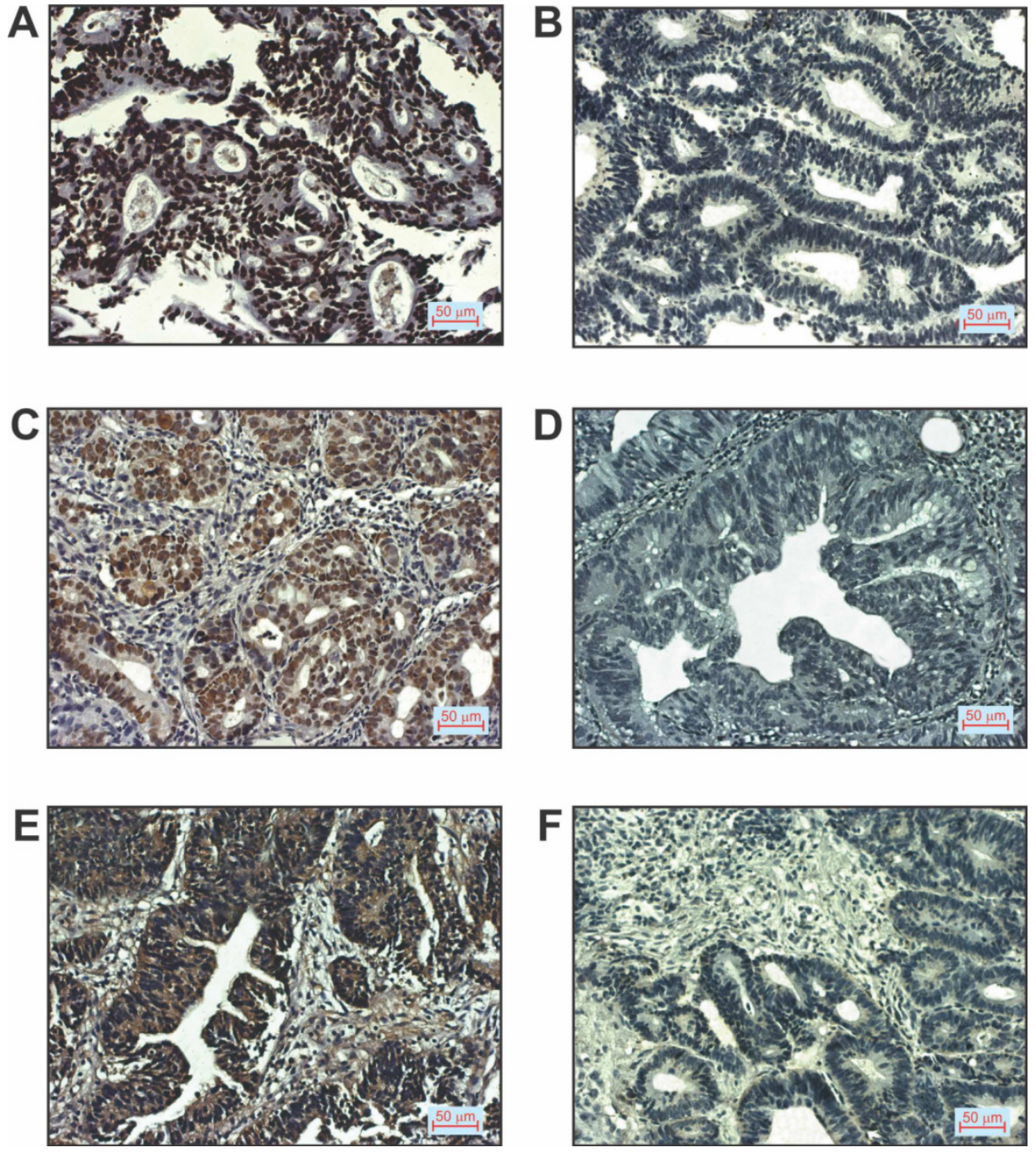

Figure 3. p 16, p53 and VEGF expression in patients with CRC. A. Positive pl6staining (200x magnification), B. Negative pl 6staining (200x magnification), C. Positive p53 staining (200x magnification), D. Negative p53 staining (200x magnification), E. Positive VEGF staining (200x magnification), F. Negative VEGF staining (200x magnification).

We have determined serum concentrations of cytokines in group of patients with CRC, UC and healthy controls. Concentration of IL-4 was significantly higher in patients with $U C$ in comparison to patients with CRC ( $p=0.014$; Fig $5 B$, left panel), while serum level of IL-17 was significantly higher in patients with $C R C(p=0.002$; Fig $5 B$, right panel). Difference in other measured cytokines between patients with CRC and patients with UC did not reached statistical significance (not shown).

\section{Logistic regression analyses of $\mathrm{p} 53$ expression and IL-4 serum level in colorectal inflammed and cancer tissue}

Binary logistic regression showed that higher expression of p53 strongly correlated with presence of CRC. Analysis showed that p53 can be a valuable marker for differentiating CRC from UC (sensitivity $60.6 \%$, specificity $95.2 \%$, cut-off $>0$; Fig 5 C, right pan- el). Analysis also showed that IL-4 (sensitivity $88.7 \%$, specificity $83.8 \%$, Fig 5C, left panel) can be a valuable marker for distinguishing UC and CRC. The optimal cutoff value estimated for IL- 4 that allows the discrimination of UC from CRC patients was $20 \mathrm{pg} / \mathrm{ml}$. For this cutoff we got sensitivity $88.7 \%$ and specificity $83.8 \%$.

\section{Discussion}

In the present study, we showed predomination of pro-inflammatory cytokines in patients with UC with presence of extraintestinal manifestations and complications, respectively and higher systemic values of TNF- $\alpha$ and IL-6 in UC patients with positive staining of p16, p53 and VEGF. Unlike UC patients, in CRC patients with positive staining of VEGF we found decreased systemic values of pro-inflammatory and increased values of immunosuppressive IL-10. We showed that higher frequency of patients with 
CRC had positive staining of p16, p53 and VEGF, in comparison to patients with UC and increased concentration of IL-4 in patients with UC in comparison to patients with CRC. Furthermore, the expression of p53 is a highly sensitive and specific marker in differentiation between UC and CRC.
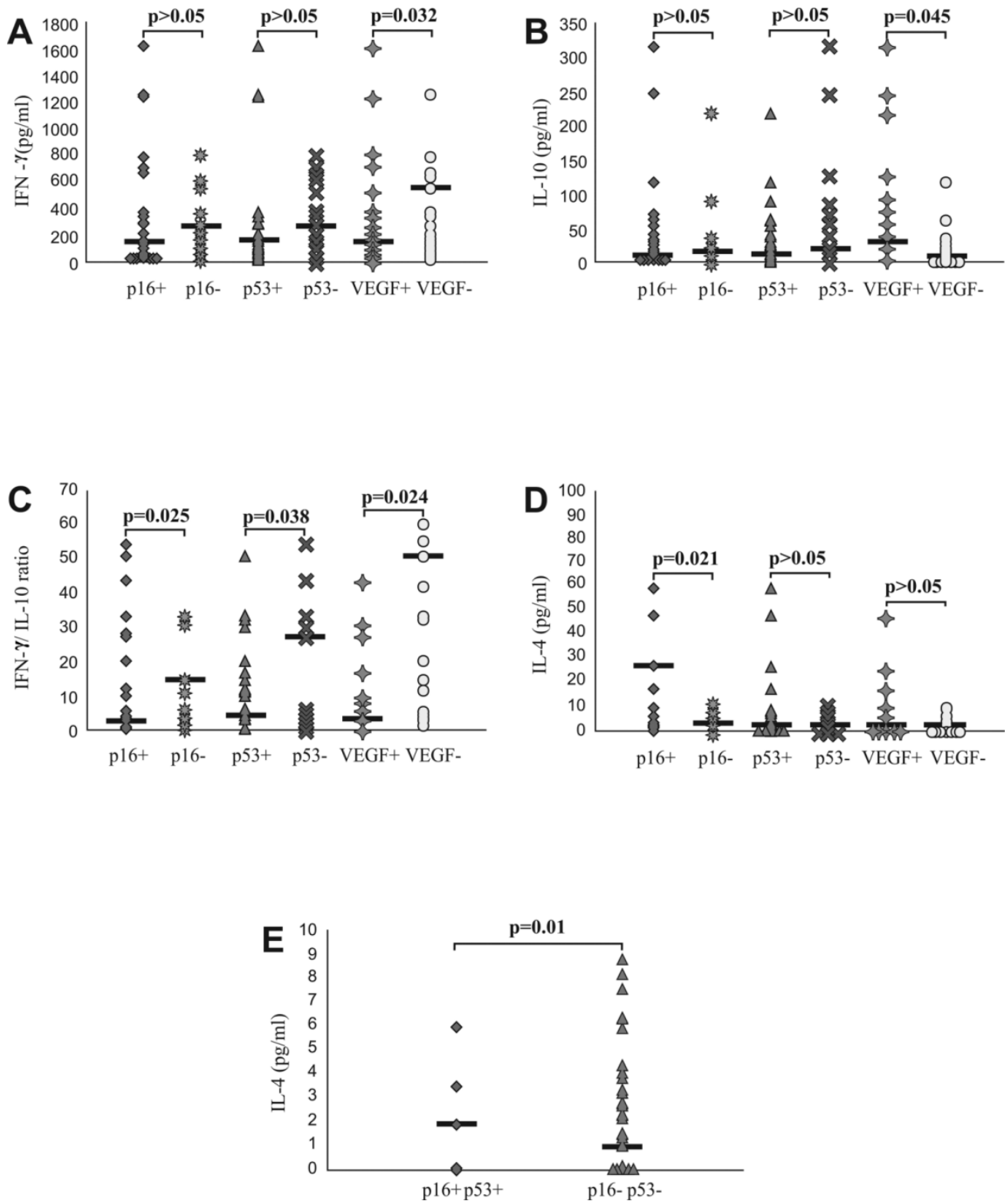

Figure 4. Systemic values of IFN-y, IL-4, IL-I0 and IFN-y/IL-I 0 ratio in patients with CRC, based on expression of p I6, p53, VEGF in affected tissue. A and B. Decreased concentration of IFN- $y$ and increased concentration of IL- 10 in patients with positive staining of VEGF. Patients with CRC were divided in two groups, based on expression of p 16, p53 and VEGF, respectively. C. Decreased IFN-y/IL- 10 ratio in patients with positive staining of p I6, p53 and VEGF, respectively. IFN-y/IL-I0 ratio was evaluated for each patient, separately. D and E. Increased concentration of IL-4 in patients with positive staining ofp 16 and simultaneous expression of 16 and p53. Patients with CRC were divided in two groups, based on expression of p I6, p53 and VEGF, respectively and also based on simultaneous expression of pl6 and p53. Statistical significance was tested by Mann-Whitney Rank Sum test or Independent Samples t-test, where appropriate. 

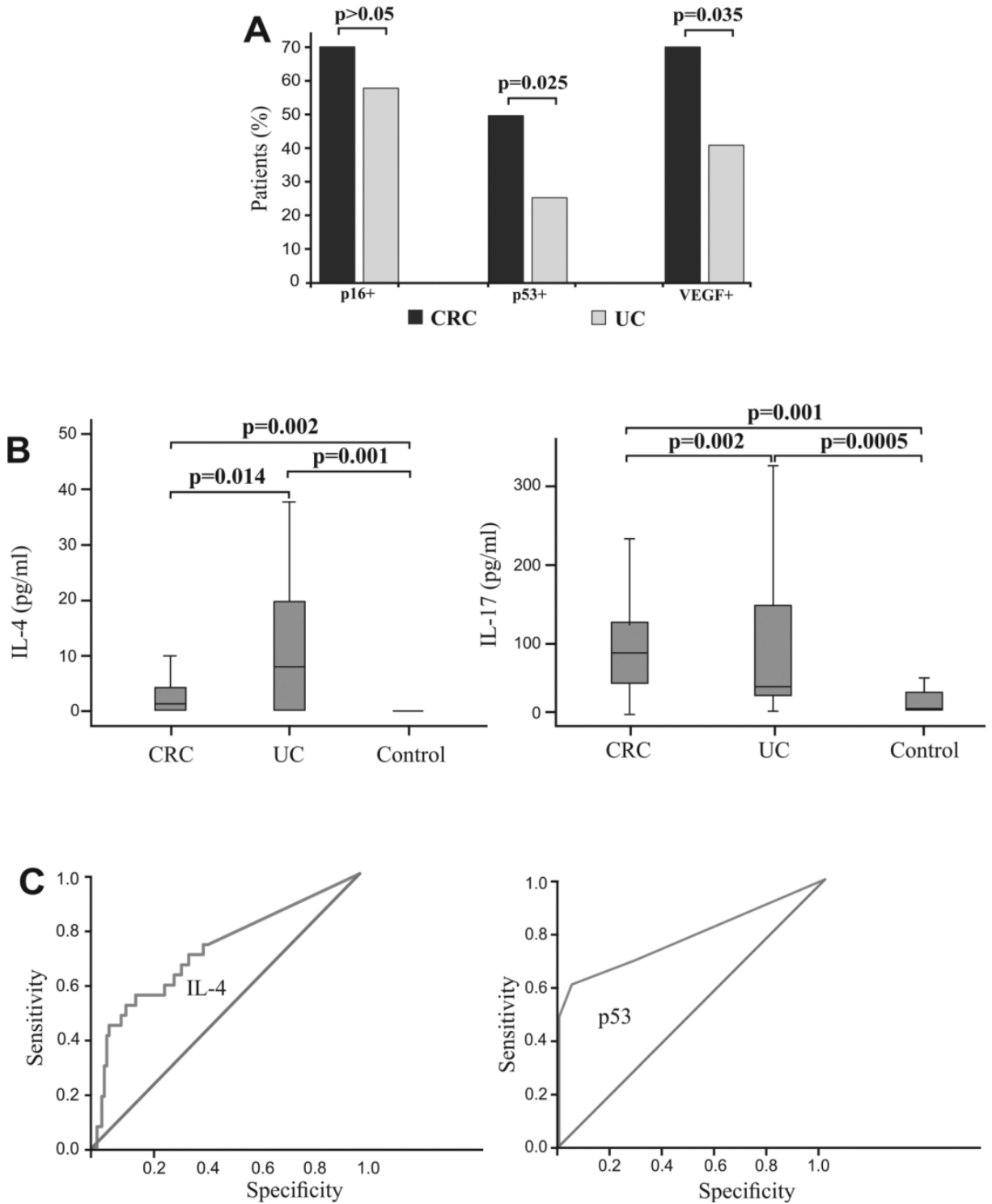

Figure 5. Expression of p16, p53 and VEGF and systemic values of IL-4 and IL-I7 in patients with CRC, with UC, and healthy controls. Specificity and sensitivity of IL-4 serum concentration and p53 expression. A. Higher percentages of CRC patients with positive staining of pI6, p53 and VEGF, respectively in comparison to patients with UC. Patients with CRC and UC were divided in two groups, respectively, based on expression of p 16, p53 and VEGF. B. Increased concentration of IL-4 in patients with UC in comparison to patients with CRC and healthy controls (left panel). Increased concentration of IL-I7 in patients with CRC in comparison to patients with UC and healthy controls (right panel). Statistical significance was tested by Mann-Whitney Rank Sum test or Independent Samples t-test, where appropriate. C. ROC curve illustrate the specificity and sensitivity of IL-4 serum concentration, comparing UC with CRC (left panel) and specificity and sensitivity of p53 expression, comparing CRC with UC (right panel).

Ulcerative colitis is inflammatory bowel disease, characterized by chronic inflammation that leads to intestinal tissue destruction. The initiation and progression of disease correlates with pro-inflammatory immune response [27]. Increased expression of pro-inflammatory cytokines TNF- $\alpha$, IL-1 $\beta$ and IL-6 and deficiency of immunosuppressive IL-10 are hallmarks of intestinal inflammation [27-29]. The obtained results in this study show that in the sera of patients with UC, a significant elevation in levels of IL-6 and IL-17/IL-10 ratio correlates with the presence of extraintestinal manifestations (Fig 2A). IL-6 can be stated as pro-inflammatory cytokine, produced by monocyte/macrophages and Th17 lymphocytes and also as an important factor for developing Th17 immune response [3]. The mechanism underlying Th17 polarization is well established. IL-6, in synergy with TGF- $\beta$ induces Th17 cells development [30]. In our study elevated IL-6 in the sera of patients with UC with the presence of extraintestinal manifestations may suggest that IL-6 is a key mediator in the development of Th17 immune response. The role of IL-6/IL-17 producing Th-17 lymphocytes in pathogenesis of Crone disease is well established [31, 32], and UC seems to be predominantly Th2 disease [33]. In line with this, we found increased serum IL-4 in UC patients, compared to healthy control (Fig 5B, left panel). Domination of Type 1 response, manifested by 
significantly lower serum levels of IL-10 and significantly higher IFN- $\gamma /$ IL-10 ratio (Fig 2B) was detected in patients with complications of disease. Also, we noticed positive correlation between the serum concentrations of TNF-a with histological grade of UC (Fig 2C), and higher concentration of CRP in patients with severe UC (not shown). These data document that relatively low serum level of IL-10 in patients with severe $\mathrm{UC}$ is insufficient to compensate secretion of IL- 6 and subsequently TNF- $\alpha$, IFN- $\gamma$ and IL-17 and enhanced type 1 and type 17 immune response. Recent publications reported increased IL-17 production in UC patients [34], and correlation between severity of disease and IL-17 produced by lipopolysaccharide stimulated peripheral blood mononuclear cells from UC patients [2].

Angiogenesis, the formation of new vasculature from an existing vascular network, plays a critical role in various pathological processes, such as tumor progression, and both acute and chronic inflammation $[17,35,36]$. It has been also suggested that this process is integral part of IBD pathology [37]. VEGF is the key mediator of angiogenesis but the role of tumor suppressor genes in angiogenesis still remains elucidated. In our study, we found higher systemic values of TNF- $\alpha$ and IL-6 in UC patients with positive staining of p16, p53 and VEGF, respectively (Fig 2D). Few studies revealed that alteration of tumor suppressor genes may contribute to neovascularization, guided by altered cytokine milieu [38, 39]. Wild type p53 down regulates VEGF expression and subsequently inhibits angiogenesis, in several tumors [40,41]. In line with this, mutant-type p53 expression correlates with VEGF in few different tumors [42, 43]. Also, wild type 16 reduce angiogenesis, via down regulation of VEGF [44] and, in rheumatoid arthritis model, p16 impairs IL-6 secretion by mononuclear cells [45]. We proposed that expressed antioncogene p16 and p53 are mutated and they are not functional [46]. Rodrugues et al. analyzed p53 cDNA by using the polymerase chain raction in 10 colorectal cancer cell lines and showed that all cell lines that expressed high levels of p53 were synthesizing mRNAs that encoded mutant p53 proteins, indicating that high expression of p53 protein correlates with the presence of p53 gene mutations in these colorectal cancer cell lines [47]. The similar results were obtained in breast cancer cell lines [48] and primary lung tumors [49]. These findings implicate that when p53 is detected immunohistologically, it is probably mutant. Lang et al. also reported that $65 \%$ of patients with overexpression of p16 in squamous cell carcinoma of the head and neck showed p16-specific gene alterations [50]. These antioncogenes may induce VEGF over expression and stimulate angiogenesis. Positive staining of p16 and
VEGF was detected in significantly higher percentages of UC patients with histological grade III (Table 1 ), indicating that disease progression is followed by higher expression of antioncogenes and subsequently VEGF. Besides its well-known role in angiogenesis, it has been suggested that VEGF also have a role in inflammation and immunity. For example, the study of Kim YS et al [51] revealed that VEGF is key mediator in the development of $\mathrm{T}$ cell priming in the airways. Their results suggest that VEGF mediates the recruitment of inflammatory cells and enhances expression of co-stimulatory molecules on recruited and resident mononuclear cells, leading to up-regulation of pro-inflammatory, Th1 and Th17 cytokines. Additionally, Basu A et al [52] confirmed facilitating role of VEGF on pro-inflammatory response, by affecting memory $\mathrm{T}$ cell function.

Chronic inflammation, underlying UC, is well established risk factor for CRC development [6]. UC-associated CRC development is a multistep process starting from inflamed epithelium toward adenocarcinoma [53]. This study analyzed UC patients and patients with sporadic CRC. Although there are so many differences between UC-associated and sporadic CRCs, some similarities exist, especially in the pathogenesis [1]. For instance, alterations of tumor suppressor genes drive progression of both CRC entities. Antioncogene p53 is well established factor in both CRCs carcinogenesis [1]. In present study, most of the CRC patients had positive staining of p16, p53 and VEGF, which was not case with UC patients (Fig $5 \mathrm{~A})$. It is well established that p53 overexpression in associated with p53 gene mutation [54]. Mutated p53 has increased half-life that explains its accumulation in the tissue [55]. We proposed that expressed antioncogenes p16 and p53 are mutated and they facilitate VEGF expression, which enhances neovascularization.

A further analysis in CRC patients also revealed decreased systemic values of pro-inflammatory IFN- $\gamma$ while increased values of immunosuppressive cytokine IL10 in CRC patients with positive staining of VEGF (Fig 4A; Fig 4B). In addition, IFN- $\gamma /$ IL10 ratio was significantly lower in group of CRC patients with positive staining of p16, p53 and VEGF, respectively (Fig 4C). Our results suggest that in UC patients, enhanced systemic pro-inflammatory immune response correlates with disease severity and local tissue angiogenesis. In contrast, in CRC patients, local tissue angiogenesis is in negative correlation with systemic pro-inflammatory immune response. As we speculated before, altered antioncogenes may induce VEGF overexpression. The recent study revealed that VEGF acts immunosuppressive on lymphocyte activation and proliferation, by increasing IDO activity in den- 
dritis cells (DCs) [56]. In line with this finding, VEGF has been shown to induce accumulation of MDSC, which suppresses DCs function and inhibit potent cell-mediated anti-tumor immunity, in patients with cancer of the digestive system [57]. Thus, in patients with CRC, VEGF acts via DCs on diminished proinflammatory immune response.

We also noticed increased IL-4 serum level in CRC patients with positive staining of p16 (Fig 4D) as well as in patients with simultaneous expression of p16 and p53 (Fig 4E). Overexpression of p16 in usually associated with gene mutation and loss of function. Recent study reveals that deficiency of p16 facilitate polarization toward M2 macrophages while inhibits proinflamatory phenotype [10]. Macrophages can be categorized into two main subsets in parallel with Th1/Th2 dichotomy: M1 (classically activated) that are induced with IFN- $\gamma[58,59]$ and M2 (alternatively activated) that differentiate after stimulation with IL-4. M2 macrophages promote angiogenesis, control inflammatory response by downregulation of M1 mediated functions and play a crucial role in type 2 immune response and antitumor immunity $[58,60]$.

Serum level of IL-17 was significantly higher in patients with CRC in comparison to UC patients (Fig $5 \mathrm{~B}$, right panel). Some studyes revealed that IL-17 facilitate tumor growth and progression, by stimulating angiogenesis [60], while others show that IL-17 induce potent antitumor immune response, responsible for rejecting tumor [61]. Exact role of IL-17 in CRC still remains unclear.

The expression of studied tumor markers may be useful in the assessment of CRC. P53 expression showed a high sensitivity and specificity for CRC in differentiating from UC (Fig 5C, right panel). We also envisage the possible role of IL-4 as a biomarker in distinguishing UC from CRC and showed that increased level of IL-4 enhanced the likelihood of UC compared to CRC (Fig 5C, left panel).

In summing up, we believe that, relatively low serum level of IL-10 in patients with severe UC is insufficient to compensate secretion of IL-6 and subsequently TNF- $\alpha$, IFN- $\gamma$ and IL-17 and enhanced type 1 and type17 immune response. Mutant-type p16 and p53 induce enhanced VEGF expression that implicates angiogenesis and, also via VEGF, enhanced production of pro-inflammatory TNF- $\alpha$ and IL-6. Pro-inflammatory role of VEGF is established in inflammatory diseases [51, 52]. In CRC patients, we found enhanced expression of p16, p53 and VEGF, and VEGF seems to have different role, immunosuppressive. In line with this, VEGF inhibits potent anti-tumor immunity, in digestive system cancer patients [57]. Our findings point on dual role of antioncogene- VEGF axis on immune response in inflam- mation (UC) and tumor growth and progression (CRC).

\section{Acknowledgments}

We thank Aleksandar Ilic, Katerina Martinova, Branislav Stevanovic and Milan Milojevic for excellent technical assistance. This work was supported by Serbian Ministry of Science and Technological Development (Grants OP 175071, OP 175103 and OP 175069) and by the Faculty of Medical Sciences, University of Kragujevac, Serbia (Grants JP 09/10).

\section{Competing Interests}

The authors have declared that no competing interest exists.

\section{References}

1. Matkowskyj KA, Chen ZE, Rao MS, Yang GY. Dysplastic lesions in inflammatory bowel disease. Arch Pathol Lab Med 2013; 137: 338-350.

2. Raza A, Yousaf W, Giannella R, Shata MT. Th17 cells: interactions with predisposing factors in the immunopathogenesis of inflammatory bowel disease. Expert Rev Clin Immunol. 2012; 8: 161-8.

3. Abbas AK, Lichtman AH, Pillai S. Cellular and molecular immunology; Seventh edition. Elsevier. 2012: 225-243.

4. Seril DN, Liao J, West AB, Yang GJ. High-iron diet: foe or feat in ulcerative colitis and ulcerative colitis-associated carcinogenesis. J Clin Gastroenterol 2006; 40: 391-397.

5. Seril DN, Liao J, Yang GJ, Yang CS. Oxidative stress and ulcerative colitis-associated carcinogenesis: studies in human and animal models. Carcinogenesis 2003; 24: 353-362.

6. Yang GJ, Taboada S, Liao J. Inflammatory bowel disease: a model of chronic inflammation-induced cancer. Methods Mol Biol 2009; 511: 193-233.

7. Itzkowitz $\mathrm{SH}$ and Yio X. Inflammation and Cancer IV. Colorectal cancer in inflammatory bowel disease: the role of inflammation. Am J Physiol Gastrointest Liver Physiol 2004; 287: 7-17.

8. Debniak T, Gorski B, Huzarski T, Byrski T, Cybulski C, Mackiewicz A et al. A common variant of CDKN2A (p16) predisposes to breast cancer. J Med Genet 2005; 42: 763-765.

9. Diehl JA, Zindy F, and Sherr CJ. Inhibition of cyclin D1 phosphorylation on threonine-286 prevents its rapid degradation via the ubiquitin-proteasome pathway. Genes Dev 1997; 11: 957-972.

10. Cudejko C, Wouters K, Fuentes L, Hannou SA, Paquet C, Bantubungi K et al. p16INK4a deficiency promotes IL-4-induced polarization and inhibits proinflammatory signaling in macrophages. Blood 2011; 118: 2556-2566.

11. Harper JW, Adami GR, Wei N, Keyomarsi K, Elledge SJ. The p21 Cdk-interacting protein Cip1 is a potent inhibitor of G1 cyclin-dependent kinases. Cell 1993; 75: 805-816.

12. Brentnall TA, Crispin DA, Rabinovitch PS, Haggitt RC, Rubin CE, Stevens AC et al. Mutations in the p53 gene: an early marker of neoplastic progression in ulcerative colitis. Gastroenterology 1994; 107: 369-78.

13. Burmer GC, Rabinovitch PS, Haggitt RC, Crispin DA, Brentnall TA, Kolli VR et al. Neoplastic progression in ulcerative colitis: histology, DNA content, and loss of a p53 allele. Gastroenterology 1992; 103: 1602-10.

14. Hussain SP, Amstad P, Raja K, Ambs S, Nagashima M, Bennett WP et al. Increased p53 mutation load in noncancerous colon tissue from ulcerative colitis: a cancer-prone chronic inflammatory disease. Cancer Res 2000; 60: 3333-7.

15. Willenbucher RF, Aust DE, Chang CG, Zelman SJ, Ferrell LD, Moore DH et al. Genomic instability is an early event during the progression pathway of ulcerative-colitis-related neoplasia. Am J Pathol 1999; 154: 1825-30.

16. Brentnall TA. Molecular underpinnings of cancer in ulcerative colitis. Curr Opin Gastroenterol 2003; 19: 64-8.

17. Folkman J. Angiogenesis in cancer, vascular, rheumatoid and other disease. Nat Med 1995; 1: 27-31.

18. Chong SK, Blackshaw AJ, Boyle S, Williams CB, Walker-Smith JA. Histological diagnosis of chronic inflammatory bowel disease in childhood. Gut 1985; 26: 55-9.

19. (IARC)., International Agency for Research on Cancer. Tumours of the colon and rectum. In: Hamilton SR, Aaltonen LA, editors. Pathology and genetics. Tumours of the digestive system. Lyon: IARC Press; 2000: 110 - 1 .

20. Jovanovic I, Radosavljevic G, Mitrovic M, Lisnic Juranic V, McKenzie ANJ, Arsenijevic N et al. ST2 Deletion Enhances Innate and Acquired Immunity to Murine Mammary Carcinoma. Eur J Immunol 2011; 41: 1902-12. 
21. Radosavljevic G, Ljujic B, Jovanovic I, Srzentic Z, Pavlovic S, Zdravkovic N et al. Interleukin-17 may be a valuable serum tumour marker in patients with colorectal carcinoma. Neoplasma 2010; 57: 135-44.

22. Abrahao AC, Bonelli BV, Nunes FD, Dias EP, Cabral MG. Immunohistochemical expression of p53, p16 and hTERT in oral squamous cell carcinoma and potentially malignant disorders. Braz Oral Res. 2011; 25: 34-41.

23. Kornbluth A, Sachar DB. Ulcerative colitis practice guidelines in adults (update): American College of Gastroenterology, Practice Parameters Committee. Am J Gastroenterol 2004;99(7):1371-85.

24. Jonathan S. Levine, and Robert Burakoff. Extraintestinal manifestations of inflammatory bowel disese. Gastroenterology and hepatlogy. 2011; 7: 235-41.

25. De Dombal FT, Watts JM, Watkinson G, Goligher JC. Local Complications of Ulcerative Colitis: Stricture, Pseudopolyposis, and Carcinoma of Colon and Rectum. British medical journal. 1966; 1: 1442-7.

26. Lashner BA, Turner BC, Bostwick DG, Frank PH, Hanauer SB. Dysplasia and cancer complicating strictures in ulcerative colitis. Digestiv disease and Sciences. 1990; 35(3): 349-352.

27. Hyun JG, Mayer L. Mechanisms underlying inflammatory bowel disease. Drug discov today dis mech 2006; 3: 457-62.

28. Shih DQ, Targan SR. Immunopathogenesis of inflammatory bowel disease. World J Gastroenterol 2008; 126: 1620-1633.

29. Li MC, He SH. IL-10 and its related cytokines for treatment of inflammatory bowel disease. World J Gastroenterol 2004; 10: 620-625.

30. Zhou L, Ivanov II, Spolski R, Min R, Shenderov K, Egawa T et al. IL-6 programs $\mathrm{T}(\mathrm{H})-17$ cell differentiation by promoting sequential engagement of the IL-21 and IL-23 pathways. Nat Immunol. 2007; 8: 967-974.

31. Fujino S, Andoh A, Bamba S, Ogawa A, Hata K, Araki Y et al. Increased expression of interleukin 17 in inflammatory bowel disease. Gut 2003; 52: 65-70.

32. Yen D, Cheung J, Scheerens $\mathrm{H}$, Poulet $\mathrm{F}, \mathrm{McCl}$ anahan $\mathrm{T}$, Mckenzie B et al. IL-23 is essential for T cell-mediated colitis and promotes inflammation via IL-17 and IL-6. J Clin Invest 2006; 116: 1310-1316.

33. Fuss IJ, Heller F, Boirivant M, Leon F, Yoshida M, Fichtner-Feigl S et al. Nonclassical CD1d-restricted NK T cells that produce IL-13 characterize an atypical Th2 response in ulcerative colitis. J Clin Invest 2004; 113: 1490-1497.

34. Kobayashi T, Okamoto S, Hisamatsu T, Kamada N, Chinen H, Saito R et al. IL23 differentially regulates the Th1/Th17 balance in ulcerative colitis and Crohn's disease. Gut 2008; 57: 1682-1689.

35. Folkman J. Angiogenesis: an organizing principle for drug discovery? Nat Rev Drug Discov 2007;6: 273-286.

36. Folkman J. Angiogenesis. Annu Rev Med 2006; 57: 1-18.

37. Chidlow JH Jr, Shukla D, Grisham MB, Kevil CG. Pathogenic angiogenesis in IBD and experimental colitis: new ideas and therapeutic avenues. Am J Physiol Gastrointest Liver Physiol 2007;293(1):G5-G18.

38. Hsu SC, Volpert OV, Steck PA, Mikkelsen T, Polverini PJ, Rao S et al. Inhibition of angiogenesis in human glioblastomas by chromosome 10 induction of thrombospondin-1. Cancer Res 1996; 56: 5684-5691.

39. Van Meir EG, Polverini PJ, Chazin VR, Su Huang HJ, de Tribolet N, Cavenee WK. Release of an inhibitor of angiogenesis upon induction of wild typep 53 expression in glioblastoma cells. Nat. Genet. 1994; 8: 171-176.

40. Mukhopadhyay D, Tsiokas L and Sukhatme VP. Wild-type p53 and v-Src exertopposing influences on human vascular endothelial growth factor gene expression. Cancer Res 1995; 55: 6161-6165.

41. Bouvet $M$, Ellis LM, Nishizaki M, Fujiwara $T$, Liu W, Bucana CD et al. Adenovirus-mediated wild-type p53 gene transferdown-regulates vascular endothelial growth factor expression and inhibits angiogenesisin human colon cancer. Cancer Res 1998; 58: 2288-2292.

42. Fontanini G, Boldrini L, Vignati S, Chine S, Basolo F, Silvestri V et al. Bcl2 and p53 regulate vascularendothelial growth factor (VEGF)-mediated angiogenesis in non-small cell lungcarcinoma. Eur J Cancer 1998; 34: 718-723.

43. Crew JP, $\mathrm{O}^{\prime}$ Brien $\mathrm{T}$, Bradburn $\mathrm{M}$, Fuggle $\mathrm{S}$, Bicknell $\mathrm{R}$, Cranston $\mathrm{D}$ et al. Vascular endothelial growth factor is a predictor of relapse and stageprogression in superficial bladder cancer. Cancer Res 1997; 57: 5281-5285.

44. Harada $\mathrm{H}$, Nakagawa $\mathrm{K}$, Iwata $\mathrm{S}$, Saito $\mathrm{M}$, Kumon $\mathrm{Y}$, Sakaki $\mathrm{S}$ et al. Restoration of Wild-Type p16 Down-Regulates Vascular Endothelial Growth FactorExpression and Inhibits Angiogenesis in Human Gliomas. Canc Res 1999; 59: 3783-3789.

45. Murakami Y, Mizoguchi F, Saito T, Miyasaka N, Kohsaka H. p16(INK4a) exerts an anti-inflammatory effect through accelerated IRAK1 degradation in macrophages. J Immunol 2012; 189: 5066-5072.

46. Santra M, Mann DM, Mercer EW, Skorski T, Calabretta B and Iozzo RV. Ectopic expression of decorin protein core causes a generalized growth suppression in neoplastic cells of various histogenetic origin and requires endogenous p21, an inhibitor of cyclin-dependent kinases. J Clin Inv 1997; 100: 149-57.

47. Rodrigues NR, Rowan A, Smith MEF, Kerr IB, Bodmer WF. p53 mutations in colorectal cancer. Proc. Natl. Acad. Sci. USA 1990; 87: 7555-59.

48. Bartek J, Iggo R, Gannon J, Lane DP. Genetic and immunochemical analysis of mutant p53 in human breast cancer cell lines. Oncogene 1990; 5: 893-900.

49. Iggo R, Gatter K, Bartek J, Lane D, Harris A. Increased expression of mutant forms of p53 oncogene in primary lung cancer. Lancet 1990; 335: 675-679.

50. Lang JC, Borchers J, Danahey D, Smith S, Stover DG, Agrawal A, Malone JP, Schuller DE, Weghorst CM, Holinga AJ, Lingam K, Patel CR, Esham B.
Mutational status of overexpressed p16 in head and neck cancer: evidence for germline mutation of p16/p14ARF. Int J Oncol. 2002; 21: 401-8.

51. Kim YS, Hong SW, Choi JP, Shin TS, Moon HG, Choi EJ et al. Vascular endothelial growth factor is a key mediator in the development of $\mathrm{T}$ cell priming and its polarization to type 1 and type $17 \mathrm{~T}$ helper cells in the airways. J Immunol. 2009; 183: 5113-5120.

52. Basu A, Hoerning A, Datta D, Edelbauer M, Stack MP, Calzadilla K et al. Cutting edge: Vascular endothelial growth factor-mediated signaling in human CD45RO+ CD4+ $\mathrm{T}$ cells promotes Akt and ERK activation and costimulates IFN-gamma production. J Immunol 2010; 184: 545-549.

53. Riddell RH, Goldman H, Ransohoff DF, Appelman H, Fenoglio C, Haggitt R et al. Dysplasia in inflammatory bowel disease: standardized classification with provisional clinical applications. Hum Pathol 1983; 14: 931-968.

54. Fogt F, Zhuang Z, Poremba C, Dockhorn-Dworniczak B, Vortmeyer A. Comparison of p53 immunoexpression with allelic loss of p53 in ulcerative colitis-associated dysplasia and carcinoma. Oncol Rep 1998; 5: 477-480.

55. Finlay CA, Hinds PW, Tan TH, Eliyahu D, Oren M, Levine AJ. Activating mutations for transformation by p53 produce a gene product that forms an hsc70-p53 complex with an altered half-life. Mol Cell Biol 1988; 8: 531-539.

56. Marti LC, Pavon L, Severino P, Sibov T, Guilhen D, Moreira-Filho CA. Vascular endothelial growth factor-A enhances indoleamine 2,3-dioxygenase expression by dendritic cells and subsequently impacts lymphocyte proliferation. Mem Inst Oswaldo Cruz 2013; 1: 1-10.

57. Nakamura I, Shibata $\mathrm{M}$, Gonda $\mathrm{K}$, Yazawa $\mathrm{T}$, Shimura $\mathrm{T}$, Anazawa $\mathrm{T}$ et al. Serum levels of vascular endothelial growth factor are increased and correlate with malnutrition, immunosuppression involving MDSCs and systemic inflammation in patients with cancer of the digestive system. Oncol Lett 2013; 5: $1682-1686$

58. Sica A, Larghi P, Mancino A, Rubino L, Porta C, Totaro MG et al. Macrophage polarization in tumour progression. Semin Cancer Biol 2008; 18: 349-355.

59. Jovanovic IP, Pejnovic NN, Radosavljevic GD, Arsenijevic NN, Lukic ML. IL-33/ST2 axis in innate and acquired immunity to tumors. Oncoimmunology 2012; 1 : 229-231.

60. Numasaki M, Fukushi J, Ono M, Narula SK, Zavodny PI, Kudo T et al. Interleukin-17 promotes angiogenesis and tumor growth. Blood 2003; 101: 2620-2627.

61. Benchetrit F, Ciree A, Vives V, Warnier G, Gey A, Sautès-Fridman C et al. Interleukin-17 inhibits tumor cell growth by means of a T-cell-dependent mechanism. Blood 2002; 99: 2114-2121. 